\title{
Benefits of short inspiratory muscle training on exercise capacity, dyspnea, and inspiratory fraction in COPD patients
}

\author{
Barakat Shahin' \\ Michele Germain ${ }^{2}$ \\ Alzahouri Kazem ${ }^{3}$ \\ Guy Annat ${ }^{4}$ \\ 'Department of Physiology, University \\ of Claude Bernard Lyon I, Lyon, \\ France; ${ }^{2}$ Chef of the Service of \\ EFR, Hospital of the Croix-Rousse \\ at Lyon, France; ${ }^{3}$ Department of \\ Medical Informatics, Hospital of St. \\ Julien, Nancy, France; ${ }^{4}$ Department \\ of Physiology, UFR Médecine Lyon \\ Grange-Blanche Université Claude \\ Bernard Lyon I, INSERM ESPRI ERI 22, \\ Lyon, France
}

\begin{abstract}
Static lung hyperinflation has important clinical consequences in patients with chronic obstructive pulmonary disease (COPD). Given that most of these patients have respiratory and peripheral muscle weakness, dyspnea and functional exercise capacity may improve as a result of inspiratory muscle training (IMT). The present study is designed to investigate the benefits of a short outpatient program of IMT on inspiratory muscle performance, exercise capacity, perception of dyspnea, and the inspiratory fraction (IF). Thirty patients ( 24 males, 6 females) with significant COPD (forced expiratory volume in one second $\left[\mathrm{FEV}_{1}\right]=46.21 \% \pm 6.7 \%$ predicted, $\mathrm{FEV}_{1}=33.6 \% \pm 8.04 \%$ predicted) were recruited for this study and had 3 months of IMT (30 minutes/day for 6 days/week) in an outpatient clinic. Following IMT, there was a statistically significant increase in inspiratory muscle performance (an increase of the maximal inspiratory pressure from $59 \% \pm 19.1 \%$ to $79 \% \pm 21.85 \%$ predicted; $p=0.0342$ ), a decrease in dyspnea (from $5.8 \pm 0.78$ to $1.9 \pm 0.57 ; p=0.0001$ ), an increase in the distance walked during the 6 minute walk test, from $245 \pm 52.37 \mathrm{~m}$ to $302 \pm 41.30 \mathrm{~m}$, and finally an increase in the IF (the new prognostic factor in COPD) from $27.6 \pm 9.7 \%$ to $31.4 \% \pm 9.8 \%$. The present study concludes that in patients with significant COPD, IMT results in improvement in performance, exercise capacity, sensation of dyspnea, and moreover an improvement in the IF prognostic factor.
\end{abstract}

Keywords: inspiratory muscle training, dyspnea, inspiratory fraction

\section{Introduction}

Chronic obstructive pulmonary disease (COPD) is defined as a preventable and treatable disease with some significant extrapulmonary effects that may contribute to the severity in individual patients. Its pulmonary component is characterized by an airflow limitation that is not fully reversible. The airflow limitation is usually progressive, and associated with an abnormal inflammatory response of the lung to noxious particles or gases (Celli and McNee 2004; GOLD 2006). This disease has a significant impact on the quality of life for patients and their families, and kills millions of people worldwide (Celli and McNee 2004; Strong et al 2005; GOLD 2006). In 2004, the American Thoracic Society/European Respiratory Society guidelines introduced into its definition the concept that, although COPD affects the lungs, it also produces significant systemic consequences (Celli and McNee 2004).

Patients with significant COPD have respiratory and peripheral muscle weakness (Gosselink et al 2000). Respiratory muscle weakness may contribute to dyspnea and to poor exercise performance (Pardy 1984; Rochester et al 1985), and for this reason, those patients must breathe at high lung volumes to maintain patency of their narrowed airways. The major abnormality of respiratory muscle function in these patients is thought to be the mechanical disadvantage caused by the hyperinflation, which depresses 
the dome of the diaphragm, shortens its fibers, and forces it to work on an ineffective portion of its length/tension curve.

In other words, both the loss of the lung's elastic recoil, and the development of expiratory flow limitations promote progressive air trapping, with an increase in the end-expiratory lung volume and a decrease in inspiratory capacity (IC). Hence, this static lung hyperinflation, and its increase during exercise (dynamic hyperinflation), have been associated with limitations in the functional capacity of those patients (Diaz et al 2000; O'Donnell et al 2001). Therefore, we believed ventilatory muscle training in these patients could be beneficial in enhancing respiratory muscle function and potentially reducing the severity of breathlessness, leading to improved exercise tolerance.

The present study was performed in order to investigate the effect of a short term program of IMT (inspiratory muscle training) on inspiratory muscle performance, the perception of dyspnea, and also its effect on the prognostic index (inspiratory fraction) in patients with significant COPD. We worked with the inspiratory fraction (the ratio of IC to total lung capacity [TLC]), which is a noninvasive test that can be easily measured in a lung function laboratory (Oga et al 2003). This fraction reflects not only the degree of lung hyperinflation, but also the functional reserve in patients with COPD (Casanova et al 2005).

\section{Patients and methods}

\section{Subjects}

Our study consisted of 30 patients ( 24 males and 6 females) of moderate and severe COPD, with spirometric evidence of significant chronic air-flow limitation (forced expiratory volume in one second $\left(\mathrm{FEV}_{1}\right)<50 \%$ predicted, $\mathrm{FEV}_{1} /$ forced vital capacity $[\mathrm{FVC}]<70 \%$ predicted), who were diagnosed as having COPD according to the criteria of the American Thoracic Society (1995), and were recruited from an outpatient clinic for the study. Thirty eight patients were invited to participate in the study, but only thirty patients had agreed to participate, and of those subjects, 3 patients were eventually excluded.

Subjects were observed during a 4-week run-in period, with their regular treatment maintained to verify stability in their clinical and functional status. This relatively stable state means that our patients were out of exacerbation of COPD or hospitalization for this period. Their characteristics are summarized in Table 1. Patients with cardiac disease or poor compliance were excluded from the study; 3 potential patients were excluded out of the study due to their cardiac problems.
Table I Anthropometric, pulmonary, and respiratory muscle data at baseline of 30 COPD patients

\begin{tabular}{lll}
\hline & Study entry & $\begin{array}{l}\text { Beginning } \\
\text { of IMT* }\end{array}$ \\
\hline Subjects $n$ & 30 & 27 \\
Age yr & $61 \pm 8.87$ yrs & $61 \pm 8.87$ yrs \\
Sex M/F & $24 / 6$ & $21 / 6$ \\
Smoking/ nonsmoking & $26 / 4$ & $23 / 4$ \\
BMI kg, m & $24 \pm 0.9$ & $24 \pm 0.9$ \\
FEV,\% predicted & $33.8 \% \pm 7.69 \%$ & $33.6 \% \pm 8.04 \%$ \\
FVC\% predicted & $73.6 \% \pm 14.3 \%$ & $72.7 \% \pm 17.7 \%$ \\
FEV/FVC\% predicted & $45.92 \% \pm 7.1 \%$ & $46.21 \% \pm 6.7 \%$ \\
TLC\% predicted & $115.8 \%+1-4.6 \%$ & $116 \%+1-3 \%$ \\
IF\% predicted & $32.1 \% \pm 4.3 \%$ & $32.02 \%+1-5.1 \%$ \\
PI max\% predicted & $59.2 \%+1-17.3 \%$ & $59 \% \pm 19.1 \%$ \\
\hline
\end{tabular}

Notes: *There was a period of four weeks of run-in between the study entry and the beginning of IMT.

Abbreviations: BMI, body mass index; IMT, inspiratory muscle training; COPD, chronic obstructive pulmonary disease; $\mathrm{FEV}_{1}$, forced expiratory volume in the first second; $\mathrm{FEV}_{1} /$ FVC, Tiffeneau index; FVC, forced vital capacity; PI max, maximal inspiratory pressure; IF, inspiratory fraction.

\section{Study design}

All tests were performed before the training and within 1 week after its completion. After this, 27 subjects were assigned to receive specific inspiratory muscle training (SIMT). The program consists of two simultaneous parts: IMT at home for a period of $30 \mathrm{~min} /$ day for six days a week, and an outpatient rehabilitation program with three times weekly education and bicycle training. In this second part, patients underwent 30 minutes of exercise and education and a 30 minute session of cycling on an ergometric bicycle.

With all patients, we performed several practice tests before obtaining the baseline value in order to correct possible training and learning effects. All data were collected by the same investigator, who was blinded to the patients and to the mode of treatment. The study protocol was approved by the institutional ethics committee, and informed consent was obtained from all the participants.

\section{Tests}

\section{Spirometry}

The FVC, FEV 1 , IC and TLC were measured three times on a computerized spirometer (MS MediSoft Module 5500; USA) and the results of the best trial are reported.

The IC was measured according to the protocol described by O'Donnell and Webb (1993), and we used the ratio (IC/TLC: Inspiratory Fraction) as a measure of resting hyperinflation. 


\section{6 minute walk test (6 MWT)}

The distance that the patient was able to walk in 6 min was determined in a measured corridor, as described for the 12-min walk test by McGavin and coworkers (1976). The patients were instructed to walk at their fastest speed and to cover the longest possible distance over 6 min under the supervision of a physiotherapist. The test was performed twice, and the best result is reported.

\section{Inspiratory muscle strength}

Inspiratory muscle strength was assessed by measuring the maximal inspiratory pressure (PI max) at residual volume and total lung capacity, respectively, as previously described by Black and Hyatt (1969). The value obtained from the best of at least three efforts was used. IMT was performed using a threshold muscle trainer (Threshold Inspiratory Muscle Trainer, Health-Scan; USA).

\section{Dyspnea}

Dyspnea was assessed using a modified Borg scale (el-Manshawi et al 1986), which is a scale of numbers ranking the magnitude of difficulty in breathing ranging from 0 (non) to 10 (maximal). Measurement of dyspnea was taken place daily at the end of 30 minutes of IMT.

\section{Training protocol}

Subjects trained daily, six times a week, with each session consisting of 30 minutes. There were no fixed number of repetitions of training for all patients, but they were requested to achieve as much training as possible during each session of IMT. Training lasted for three months, and was performed using a threshold inspiratory trainer. The subjects started breathing at a resistance equal to $15 \%$ of their PI max for three days. The resistance was then increased incrementally by $10 \%$ each two days, to reach $60 \%$ of their PI max at the end of the first two weeks of training. SIMT was then continued at $60 \%$ of the PI max until the end of the training period.

\section{Data analysis}

The results are expressed as the mean \pm standard deviation (SD). Comparisons of lung function, respiratory muscle strength and endurance, the results of the $6 \mathrm{MWT}$, and the rating of dyspnea within the group was carried out using the two-way repeated-measures analysis of variance.

\section{Results}

All values are expressed as mean \pm SD unless stated otherwise. Table 1 shows the relevant anthropometric and lung function characteristics of the $27 \mathrm{COPD}$ patients. There were no significant differences between the patients for age, body mass index (BMI), mean $\mathrm{FEV}_{1}$ and FVC, PI max, dyspnea, IF, and the 6 MWT at the beginning of the study; in other words, all patients initially had approximately the same scores for these parameters.

\section{Spirometry}

Following the IMT period, there was no significant change in the $\mathrm{FEV}_{1}$ or in the $\mathrm{FVC}$ in the group of patients as shown in Table 2 .

\section{Inspiratory muscle strength}

Following the training period there was a statistically significant increase in the PI $\max$ in most of our patients, from $40 \mathrm{~cm} \pm 3.6 \mathrm{~cm}$ to $54 \mathrm{~cm} \pm 3.9 \mathrm{~cm} \mathrm{H}^{2} \mathrm{o} ; p<0.005$. There was also a statistically significant increase in the PI max predicted values, from $59 \% \pm 19.1 \%$ to $79 \pm 21.85 \%$; $p=0.0342$ (Table 2).

\section{WMT}

There was a statistically significant improvement in the distance walked during the $6 \mathrm{MWT}$ following the training period in most of the patients, from $245.90 \mathrm{~m} \pm 52.37 \mathrm{~m}$ to $302.4 \mathrm{~m}$ $\pm 41.30 \mathrm{~m} ; p=0.0054)$. This increase of $57 \mathrm{~m}$ in the mean distance walked in 6 minutes is shown in Table 2.

\section{Dyspnea}

A statistically significant decrease in the mean Borg score of dyspnea during breathing against resistance was associated with an increase in inspiratory muscle strength in the group of patients ( $5.8 \pm 0.78$ to $1.9 \pm 0.57 ; p=0.0001)$ (Table 2$)$.

\section{Inspiratory fraction}

An increase, but not statistically significant, in the ratio $\mathrm{IF}=\mathrm{IC} /$ TLC was noted following the inspiratory training period (from $27.6 \% \pm 9.7 \%$ to $31.4 \% \pm 9.8 \% ; p=0.4$ ) (Table 2 ).

Table 2 Values* at baseline and following training for pulmonary function, muscle data, IF, dyspnea, and distance of 6 MWT

\begin{tabular}{llll}
\hline & Baseline & After SIMT & p value \\
\hline FEV \% predicted & $33.6 \pm 8.04$ & $33.9 \pm 9.6$ & 0.849 \\
FVC\% predicted & $72.7 \pm 17.7$ & $71.80 \pm 18.27$ & 0.93 \\
FEV $/$ /FVC\% predicted & $46.21 \pm 6.7$ & $47.2 \pm 5.6$ & 0.87 \\
IF\% predicted & $27.6 \pm 9.7$ & $31.4 \pm 9.8$ & 0.40 \\
PI max\% predicted & $59 \pm 19.10$ & $79 \pm 21.85$ & $0.0342^{\dagger}$ \\
Dyspnea & $5.8 \pm 0.78$ & $1.9 \pm 0.567$ & $0.000 \mathrm{I}^{\dagger}$ \\
6 MWT & $245.9 \pm 52.37$ & $302.4 \pm 41.3$ & $0.0054^{\dagger}$ \\
\hline
\end{tabular}

Notes: *Values are expressed as mean \pm SD. tStatistically significant.

Abbreviations: 6 MWT, 6 minute walk test; SD, standard deviation; SIMT, specified inspiratory muscle training. 


\section{Discussion}

To our knowledge, this is the first study to use the ratio IC/ TLC (IF), which is an independent predictor of all-cause and respiratory mortality in patients with COPD, in order to evaluate the outcome of an outpatient IMT in those patients.

This study shows that in patients with significant COPD, inspiratory muscles can be trained with improvement of muscle strength, as measured by the increasing of PI max. The improvement in the inspiratory muscle performance is associated with improved exercise performance and in the sensation of dyspnea in daily activities, with no improvement in $\mathrm{FEV}_{1}$ or FVC.

Respiratory muscles in COPD have considerably different loading patterns than other skeletal muscles. In limb muscles, especially lower-extremity muscles, muscle weakness and respiratory insufficiency lead to inactivity and chronic underloading of the muscles. In contrast, respiratory muscles have to deal with an increased workload to breathe, and are thus chronically overloaded (MacIntyre 2006). These muscles are also compromised by hyperinflation, produced by airway collapse and low elastic recoil (Similowski et al 1991; O’Donnell 2002).

The scientific evidence about IMT at the present time is somewhat controversial. However, in a meta-analysis of 17 articles on SIMT (Smith et al 1992), it has been shown that when the training stimulus was adequate to induce a significant improvement in respiratory muscle performance there was a significant reduction in the severity of dyspnea and an improved functional exercise capacity.

In the present study, it has been demonstrated that the improvement in the inspiratory muscle performance was associated with an increase in the 6 MWT distance and in the sensation of dyspnea during breathing against resistance. Although this increase may possibly be influenced by the accompanying outpatient program, but our results were obtained after the end of our IMT program, and we did not wait until the end of the accompanying program. Such results parallel other studies, in which significantly increased inspiratory muscle performance was associated with improved exercise tolerance and decreased dyspnea (Lotters et al 2002).

The perception of dyspnea is critical, but it presents a paradox to patients with airway obstruction. It limits daily activity and impairs quality of life and provides a warning of deterioration.

It has been shown that there is a close relationship between the sensation of breathlessness and respiratory muscle force both in healthy subjects and in patients with
COPD who have severe lung function impairment (Patessio et al 1989). The respiratory muscles, like other skeletal muscles, can be trained (Pardy and Leith 1984), resulting in a significant improvement in respiratory muscle performance. This increase in respiratory muscle performance was associated with a decrease in the sensation of breathlessness in patients who had COPD with a pretraining respiratory muscle weakness (Patessio et al 1989).

We utilized the IF, which reflects not only the degree of lung hyperinflation but also the functional reserve in patients with COPD (Casanova et al 2005). Indeed, it is a noninvasive test that can be easily measured in a lung function laboratory (Oga et al 2003; Marquis 2002). It provides a descriptor of the severity of lung impairment and is thus an independent and excellent predictor of respiratory-related and other mortalities in COPD. The $25 \%$ IC/TLC value offers the best combined sensitivity, specificity, positive and negative predictive value (Casanova et al 2005). The use of this index in our study with its improvement (from $27 \%$ to $31 \%$ ) affords a good idea of the positive effect of our IMT in the life of COPD patients.

Therefore, our present study contributes additional information to the knowledge regarding the benefits of IMT on exercise capacity and dyspnea in daily activities. It supports previous data that an outpatient program can provide benefits to patients with COPD.

A second important outcome of the current study, although comparison with an inpatient program was not made, is that IMT in patients with significant COPD may be performed at home, under some supervision, with relatively good compliance.

The third important finding of the current study is the use of the IF for evaluating the outcome of the program which measured the resting hyperinflation in patients with COPD and predicted the mortality of all causes in these groups of patients.

However, this study had some limitations. First, there was no control group. This was because the study was not designed to compare the outcome of an outpatient with an inpatient program, or to compare inspiratory with expiratory muscle training. Secondly, few women were included. This low number probably reflects the problem of underdiagnosis of COPD in women, and possibly the relatively late beginning of smoking among women. Third, we would have liked to follow our patients for a longer period. Fourth, the size of our population was relatively small, which affected the significance of our results.

Finally, the current study was not designed to determine whether domiciliary IMT reduces exacerbations and 
hospital admissions in COPD patients. Further studies should be performed in order to overcome these problems and limitations.

\section{Disclosure}

The authors report no conflicts of interest.

\section{References}

American Thoracic Society. 1995. Standard for diagnosis and care of patients with COPD. Am J Respir Crit Care Med, 152:78-121.

Black LF, Hyatt RE. 1969. Maximal respiratory pressures: normal values and relationship to age and sex. Am Rev Respir Dis, 99:696-702.

Casanova C, Cote C, de Torres JP, et al. 2005. Inspiratory-to-total lung capacity ratio predicted mortality in patients with chronic obstructive pulmonary disease. Am J Respir Crit Care Med, 171:591-7.

Celli BR, MacNee W. 2004. Standard for diagnosis and treatment of patients with COPD: a summary of the ATS/ERS position paper. Eur Respir $J$, 23:932-46.

Diaz O, Villafrance C, Ghezzo H, et al. 2000. Role of inspiratory capacity on exercise tolerance in COPD patients with and without expiratory flow limitation at rest. Eur Respir J, 16:269-75.

El-Manshawi A, Killian KJ, Summers E, et al. 1986. Breathlessness during exercise with and without resistive load. J Appl Physiol, 61:896-905.

[GOLD] Global Initiative for Chronic Obstructive Lung Disease. 2006. Workshop Report: Global strategy for diagnosis, management, and prevention of COPD. Available from http://www.goldcopd.com/.

Gosselink R, Toosters T, Decramer M. 2000. Distribution of muscle weakness in patients with stable chronic obstructive pulmonary disease. J Cardiopulm Rehabil, 20:353-60.

Lotters F, Van Tol B, Kwakkel G, et al. 2002. Effects of controlled inspiratory muscle training in patients with COPD: a meta analysis. Eur Respir J, 20:570-6.
MacIntyre NR. 2006. Muscle dysfunction associated with chronic obstructive pulmonary disease. Respir Care, 51:840-8.

Marquis K, Debigare R, Lacasse Y, et al. 2002. Midthigh muscle cross sectional area is a better predictor of mortality than body mass index in patients with chronic obstructive pulmonary disease. Am J Respir Crit Care Med, 166:809-13.

McGavin CR, Gupta SP, McHardy GJR. 1976. Twelve-minute walking test for assessing disability in chronic bronchitis. BMJ, 1:822-3.

O'Donnell DE, Revill SM, Webb KA. 2001. Dynamic hyperinflation and exercise intolerance in chronic obstructive pulmonary disease. Am J Respir Crit Care Med, 164:770-7.

O'Donnell DE, Web KA. 1993. Exertional breathlessness in patients with chronic airflow limitation: the role of lung hyperinflation. Am Rev Respir Dis, 148:1351-7.

O'Donnell DE. 2002. Exercise limitation and clinical exercise testing in chronic obstructive pulmonary disease. In: Weisman IM, Zeballos RJ, (eds). Progress in respiratory research: Clinical exercise testing. Basel: Karger, pp. 138-58.

Oga T, Nishimura K, Tsukino M et al. 2003. Analysis of the factor related to mortality in chronic obstructive pulmonary disease. Am J Respir Crit Care Med, 167:544-9.

Pardy RL, Leith DE. 1984. Ventilatory muscle training. Respir Care, 29:278-84.

Patessio A, Rampulla C, Fracchia C, et al. 1989. Relationship between the perception of breathlessness and inspiratory resistive loading: report on a clinical trial. Eur Respir J, 2:587-91.

Rochester DF, Braun NT. 1985. Determinants of maximal inspiratory pressure in chronic obstructive pulmonary disease. Am Rev Respir Dis, 132:42-7.

Similowski T, Yan S, Gauthier AP, et al. 1991. Contractile properties of the human diaphragm during chronic hyperinflation. N Engl J Med, 325:917-23.

Smith K, Cook D, Guyatt GH, et al. 1992. Respiratory muscle training in chronic airflow limitation: a meta - analysis. Am Rev Respir Dis, 145:533-9.

Strong K, Mathers C, Leeder S. 2005. Preventing chronic diseases: how many lives can we save? Lancet, 366:1578-82. 
